\title{
Malignant middle-cerebral artery territory infarction in tuberculous vasculitis
}

\author{
Salvadeeswaran Meenakshi-Sundaram ${ }^{1}$, Sundararajan Srinivasan ${ }^{1}$, Somalinga Nagendran Karthik ${ }^{1}$, \\ Suresh Pandi ${ }^{1}$, Alagappan Periyakaruppan ${ }^{2}$, Bharathi Sundar ${ }^{1}$ \\ ${ }^{1}$ Department of Neurosciences, Apollo Speciality Hospitals, Madurai 625020, Tamil Nadu, India. \\ ${ }^{2}$ Department of Radiology, Apollo Speciality Hospitals, Madurai 625020, Tamil Nadu, India.
}

\section{A B S T R A C T}

Intracranial large vessel involvement is an unusual complication of tuberculous meningitis. The authors report a 39-year-old female presenting with an episode of seizure, followed by rapid decline in sensorium without prominent systemic features. An initial cranial magnetic resonance imaging revealed tuberculomata and patchy infarcts. Despite antituberculous therapy, she progressively worsened. A cranial computed tomography scan done following the worsening revealed a massive middle-cerebral artery (MCA) infarct. Unfortunately, the patient died in spite of decompressive craniotomy. Malignant MCA territory infarct is a rare and potentially fatal complication of tuberculous meningitis.

Key words: Arteritis, malignant middle-cerebral artery territory infarct, tuberculous meningitis

\section{INTRODUCTION}

Involvement of small and medium sized vessels of the intracranial vasculature is well recognised in tuberculosis. Large artery involvement is however a rare manifestation of tuberculous vasculitis. Here, we present the first reported case in the literature of a patient who presented with a malignant middlecerebral artery (MCA) territory infarct as a manifestation of tuberculous vasculitis.

\section{CASE REPORT}

A 39-year-old female had presented to her general practitioner 15 days prior to this admission with a history of cough with expectoration and fever. Sputum stained with Ziehl-Neelsen stain revealed the acid fast bacilli morphologically characteristic of mycobacterium tuberculosis. A few days prior to this admission, she had had an episode of generalized tonic-clonic seizure followed by progressive decline in sensorium. Cranial magnetic resonance imaging (MRI) [Figure 1a-c] done at

\begin{tabular}{|l|l|}
\hline \multicolumn{2}{|c|}{ Access this article online } \\
\hline Quick Response Code: & \\
\hline & Website: \\
\hline & www.nnjournal.net \\
\cline { 2 - 3 } & DOI: \\
\hline
\end{tabular}

the referring hospital revealed multiple heterogeneously enhancing nodular lesions of varying sizes involving right posterior parietooccipital and frontal subcortical and cortical regions with significant thickening of adjacent leptomeninges and exudates. These features were classical of focal cortical and subcortical tuberculomata with cerebritis and leptomeningitis, especially on a background of acid-fast bacilli detected in sputum. There was also cerebral edema with effacement of the ventricular system, sulci and cisterns and focal vasogenic edema around the right parieto-occipital lesion with patchy areas of diffusion restriction representing arteritis-induced-infarcts. Antituberculous drugs (isoniazid $300 \mathrm{mg} /$ day, rifampicin $600 \mathrm{mg} /$ day, pyrazinamide $1500 \mathrm{mg} /$ day and ethambutol $800 \mathrm{mg} /$ day), dexamethasone $8 \mathrm{mg}$ twice a day and phenytoin $(300 \mathrm{mg} /$ day) were administered. Sensorium had however gradually deteriorated, and she had become unresponsive since a day when she was transferred to our hospital.

On examination, she was hemodynamically stable, pyrexial and anemic. Neurologically she was comatose with decerebrate movements of right upper limb to pain. There were no meningeal signs. On the oculocephalic maneuver, there was failure of adduction of right eye and upgaze was absent. Pupils were $5 \mathrm{~mm}$ on the right side and $2 \mathrm{~mm}$ on the left side. Optic fundi were unremarkable. Tendon reflexes were brisk bilaterally, and plantars were extensors on both sides. Investigations revealed: hemoglobin

Corresponding Author: Dr. Salvadeeswaran Meenakshi-Sundaram, Department of Neurosciences, Apollo Speciality Hospitals, Lake View Road, KK Nagar, Madurai 625020, Tamil Nadu, India. E-mail: drsundarsms@gmail.com 


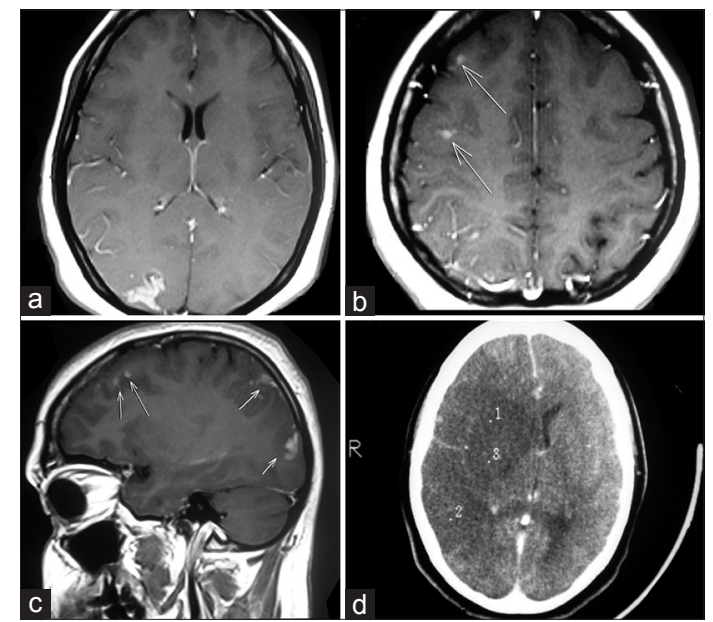

Figure 1: Tuberculous meningitis with arteritis leading to malignant middle cerebral artery (MCA) infartion. Gadolinium enhanced axial T1-weighted magnetic resonance images shows (a) enhancing leptomeningeal exudates with subcortical, juxta cortical and cortical granulomata in the right posterior parietooccipital region: (b) fronto-parietal cortical granuloma with leptomeningeal enhancement; (c) Frontotemporo-parieto-occipital cortical, juxta cortical granuloma with leptomeningeal enhancement. (d) contrast enhanced computerized tomography (CT) axial image shows malignant infarct involving the entire right MCA territory with compression of the right lateral ventricle and midline shift to the left side. One and three marked regions in CT represent basal ganglionic region and two represent temporo occipital region with no significant hemorrhagic component

$12.9 \mathrm{~g} / \mathrm{dL}$; total white cell count $14.8 \times 10^{9} / \mathrm{L}$; differential count-polymorphs $84 \%$, lymphocytes $13 \%$, myelocytes $1 \%$ and stab forms $2 \%$; platelet count $19.0 \times 10^{9} / \mathrm{L}$ and erythrocyte sedimentation rate $29 \mathrm{~mm} / \mathrm{h}$. A whole list of investigations including blood sugar, renal and liver functions, electrolytes, coagulation profile, urinalysis, human immunodeficiency virus test, antinuclear antibodies, ds-DNA, rheumatoid factor, venereal disease research laboratory, hepatitis B surface antigen, C-reactive protein, antineutophil cytoplasmic antibodies, lupus anticoagulant and antiphospholipid antibody tests were noncontributory. Cranial enhanced computed tomography scan [Figure 1d] revealed an acute nonhemorrhagic complete right MCA territory infarct and few enhancing lesions in and around the sulci in the right occipital, posterior parietal and high frontal lobes with severe right ventricular compression. Marked cerebral edema and midline shift were observed. Decompressive surgery in the form of right fronto-parieto-temporal craniectomy was done as for malignant MCA infarct. Histopathological evaluation of leptomeningeal tissue obtained during surgery revealed features of chronic meningitis with dense lymphohistiocytic infiltrate forming microgranuloma surrounding the meningeal blood vessels. Clinically she deteriorated with bilateral pupillary dilatation on day 3 of admission with hypotension. She, unfortunately, succumbed to the illness on day 4 of admission.

\section{DISCUSSION}

Tuberculous vasculitis is an important cause of stroke in the young in developing countries. Stroke in tuberculous meningitis occurs in $15-57 \%$ of patients especially in advanced stage and severe illness and are usually multiple, bilateral and located in the basal ganglia, especially the tubercular zone, which comprises of the caudate, anterior thalamus, anterior limb and genu of the internal capsule. Cortical stroke can also occur due to the involvement of the proximal portion of the middle, anterior and posterior cerebral arteries, as well as the supraclinoid portion of the internal carotid and basilar arteries., ${ }^{[1]}$ While pathological changes suggestive of intracranial vasculitis are common in tuberculosis even without corresponding clinical features, to our knowledge, this is the first reported case of malignant MCA territory infarct in tuberculous meningitis. The initial MRI features [Figure 1a-c] were fairly typical of tuberculosis, especially in the context of positive acid-fast bacilli in the sputum. In a pathological study of 23 postmortem cases of tuberculous meningitis, phlebitis was found in 22 and arteritis of varying degrees in 20. Thrombosis in the territory of MCA with infarction was seen in one of these patients. Both hemorrhagic and nonhemorrhagic infarcts were visualized. ${ }^{[2]}$ Tuberculous vasculitis usually involves vessels that traverse the basal exudates or are located within the brain parenchyma. ${ }^{[3]}$ Arteries running through the subarachnoid space may show obliterative endarteritis with inflammatory infiltrates in their walls and marked intimal thickening. ${ }^{[4]}$ Various stroke syndromes are known with involvement of different regions of the brain including basal ganglia, thalamus, cerebral hemispheres and cerebellum with varying outcomes. ${ }^{[5,6]}$ In our patient, the infarct was extensive with significant mass effect and transtentorial coning. The neuro-ophthalomological findings noted were suggestive of midbrain involvement (right pupillary mydriasis, right medial rectus involvement and paralysis of upgaze). The course of the disease was rapid and malignant despite antitubercular and steroid therapy.

Elective hemicraniectomy has been advocated as a lifesaving therapeutic option in patients with complete MCA infarction. ${ }^{[7]}$ Young age, involvement of the nondominant hemisphere and progressively worsening neurological status despite aggressive medical therapy warranted consideration of the surgical procedure. However, we were unsuccessful as the patient deteriorated and died despite aggressive treatment. Clinical deterioration despite surgery is well known to occur in malignant cerebral infarction.

In conclusion, we report a patient with malignant MCA infarct as a consequence of tuberculosis. Such a manifestation may portend a poor prognosis despite aggressive life-saving measures. 


\section{ACKNOWLEDGMENTS}

We acknowledge Dr. Krishnan Swaminathan, Consultant Endocrinologist, Apollo Speciality Hospitals, Madurai, who read the manuscript, edited it and guided us in the finalization of the manuscript.

\section{REFERENCES}

1. Misra UK, Kalita J, Maurya PK. Stroke in tuberculous meningitis. J Neurol Sci 2011;303:22-30.

2. PolteraAA. Thrombogenic intracranial vasculitis in tuberculous meningitis. A 20 year "post mortem" survey. Acta Neurol Belg 1977;77:12-24.

3. Lammie GA, Hewlett RH, Schoeman JF, Donald PR. Tuberculous cerebrovascular disease: a review. J Infect 2009;59:156-66.

4. Frosch MP, Anthony DC, Girolami U. The central nervous system. In: Kumar V, Abbas AK, Fausto N, Aster JC, editors. Robbins and Cotran Pathologic Basis of Disease. 8th ed. Philadelphia: WB Saunders Co.; 2010. p. 1279-344.
5. Javaud N, Certal RDaS, Stirnemann J, Morin AS, Chamouard JM, Augier A, Bouchaud O, Carpentier A, Dhote R, Dumas JL, Fantin B, Fain O. Tuberculous cerebral vasculitis: retrospective study of 10 cases. Eur J Intern Med 2011;22:e99-104.

6. Kalita J, Misra UK, Nair PP. Predictors of stroke and its significance in the outcome of tuberculous meningitis. J Stroke Cerebrovasc Dis 2009; $18: 251-8$

7. Jüttler E, Unterberg A, Woitzik J, Bösel J, Amiri H, Sakowitz OW, Gondan M, Schiller P, Limprecht R, Luntz S, Schneider H, Pinzer T, Hobohm C, Meixensberger J, Hacke W, DESTINY II Investigators. Hemicraniectomy in older patients with extensive middle-cerebral-artery stroke. N Engl J Med 2014;370:1091-100.

Cite this article as: Meenakshi-Sundaram S, Srinivasan S, Karthik SN, Pandi S, Periyakaruppan A, Sundar B. Malignant middle-cerebral artery territory infarction in tuberculous vasculitis. Neuroimmunol Neuroinflammation 2014;1(2):95-7.

Source of Support: Nil. Conflict of Interest: No.

Received: 14-05-2014; Accepted: 18-07-2014 